\title{
Modelo experimental de esplenectomia laparoscópica em $\operatorname{ratos}^{1}$
}

\author{
Vanessa Alves Costa ${ }^{2}$ \\ Fabrício Mascarenhas de Oliveira² \\ Lúcio Couto de Oliveira Jr² \\ Mario Castro Carreiro ${ }^{3}$ \\ Pedro Guimarães ${ }^{4}$
}

\begin{abstract}
Costa VA, Oliveira FM, Oliveira Jr LC, Carreiro MC, Guimarães P. Modelo experimental de esplenectomia laparoscópica em ratos. Acta Cir Bras [serial online] 2003 Nov-Dez;18(6). Disponível em URL: http://www.scielo.br/acb.

RESUMO - Objetivo: Desenvolver um modelo de esplenectomia laparoscópica em ratos. Métodos: Foram utilizados trinta e cinco ratos machos ( Rattus Norvegicus Albinus, linhagem Wistar), pesando 250 +/- $50 \mathrm{~g}$.Os animais foram anestesiados com cetamina e xylazina e foi estabelecido pneumoperitônio de $\mathrm{CO} 2 \mathrm{com}$ pressão de $7 \mathrm{mmHg}$, através da agulha de Veress. Após pneumoperitônio, dois trocateres de $5 \mathrm{~mm}$ e um de $11 \mathrm{~mm}$ foram alocados na parade abdominal obedecendo a triangulação. O ligamento gastroesplênico foi dissecado para mobilização do baço. Os vasos hilares foram dissecados e eletrocoagulados utilizando a pinça "Maryland" e o gancho "HooK". Um "endobag" adaptado, foi utilizado para retirar o orgão da cavidade abdominal. Resultados :Um animal $(2,86 \%)$ morreu na indução anestésica. Após um período de observação de oito dias, trinta animais sobreviveram $(85,7 \%)$ e quatro $(11,42 \%)$ Morreram no pós-operatório imediato. Em relação às complicações, Aderências intra-abdominais foram encontradas em 25,71\% dos animais (n=9). Conclusão: A esplenectomia laparoscópica é um modelo factível por conta da baixa taxa de mortalidade além do baixo custo para treinamento da técnica.
\end{abstract}

DESCRITORES - Cirurgia laparoscópica. Esplenectomia. Ratos.

\section{Introdução}

Desde o desenvolvimento da colecistectomia laparoscópica em 1987, houve entusiasmo considerável na aplicação de técnicas "minimamente invasivas" para tratar outras desordens abdominais e torácicas. A motivação para o desenvolvimento da técnica laparoscópica é prover uma alternativa segura e efetiva que reduza o desconforto e do paciente, os serviços de internamento e os custos hospitalares. ${ }^{1}$

A cirurgia experimental tem sido a referência para que novas técnicas, como a laparoscopia, sejam aperfeiçoadas ${ }^{2}$. A vídeo-cirurgia requer habilidades técnicas mais complexas: uma apurada coordenação motora, destreza para realizar movimentos de terceira dimensão num campo bidimensional e transferir a sensibilidade tátil para manipulação através do instrumental específico ${ }^{3}$. Tradicionalmente, animais de grande porte como cães e porcos são utilizados na laparoscopia experimental por apresentarem o tamanho e anatomia compatível com um homem de estatura mediana. Entretanto esses animais apresentam alto custo, dificuldade em acomodação em pequenos biotérios e são pouco utilizados em pesquisas imunológicas ${ }^{4}$. Existem trabalhos internacionais, ${ }^{2-4}$ descrevendo a técnica laparoscópica em ratos, utilizando instrumental mais acurado ao diminuto campo operatório e que por conta dessa adaptação são dispendiosos. ${ }^{7-11}$

Como não foram encontrados modelos de esplenectomia laparoscópica na literatura nacional, buscamos desenvolver um modelo que atendesse aos baixos custos, fácil manejo, utilização de material convencional de humanos adultos e que fosse útil em pesquisas imunológicas e oncológicas.

\section{Métodos}

Os experimentos foram aprovados pela Comissão de Ética da Escola Bahiana de Medicina e Saúde Pública (E.B.M.S.P. BA) e obedeceram os preceitos do Colégio Brasileiro de Experimentação Animal (COBEA).

Para o presente estudo, foram utilizados 35 ratos Rattus norvegicus albinus da linhagem Wistar, procedentes do biotério da E.B.M.S.P. - BA, pesando 250 +/- 50 $\mathrm{g}$, com idade média de 3 meses sendo todos machos. Os animais foram colocados

1. Trabalho realizado na Escola Bahiana de Medicina e Saúde Pública (EBMSP).

2. Acadêmicos de Medicina da EBMSP.

3. Professor Adjunto IV da Universidade Federal da Bahia (UFBA). Coordenador da Disciplina de Técnica Operatória e Cirurgia Experimental da UFBA.

4. Professor Adjunto da Disciplina de Técnica Operatória e Cirurgia Experimental da UFBA. Doutor pela Universidade Federal de São Paulo - Escola Paulista de Medicina (UNIFESP - EPM). 
em gaiolas individuais, medindo 20X30X40 cm, aclimatados durante 15 dias a uma temperatura de $25^{\circ} \mathrm{C}$, em períodos de 12 horas luz e 12 horas escuridão com ração e água livres. Todos os animais foram avaliados no intra-operatório quanto à presença ou não de hemorragia controlável. Após um período de 8 dias, os animais foram avaliados quanto ao peso e à presença ou ausência de: áreas de hemorragia, abscessos intraabdominais, aderências e deiscência de sutura.

\section{Pré-operatório}

Cerca de oito horas antes do procedimento os animais foram colocados em jejum, e anestesiados por via intra-peritoneal com cetamina $(7 \%)$ e xilasina $(0.3 \%)$ na proporção de 2: $1 \mathrm{~mL}$, com 0,2 mL/100 g de peso da solução. Em seguida, foram posicionados na mesa cirúrgica em decúbito dorsal, tricotomizados e submetidos a antissepsia.

\section{Ato operatório}

Após a colocação dos campos, foi feita uma incisão de $0,5 \mathrm{~cm}$ a quatro $\mathrm{cm}$ do rebordo costal direito na linha axilar anterior, e com auxílio de duas pinças Allis a agulha de Veress puncionou a cavidade abdominal. O insuflador foi programado para uma pressão máxima de $7 \mathrm{mmHg}^{6}$ e a menor velocidade de fluxo $(0,8 \mathrm{~L} / \mathrm{mim}) \mathrm{e}$ então foi acoplado à agulha de Veress após os testes de segurança. Com o pneumoperitônio estabilizado, a mesa de contenção foi inclinada $45^{\circ}$ para direita, favorecendo o acesso à loja esplênica.

A incisão da agulha de Veress foi ampliada para alojar um trocáter de $11 \mathrm{~mm}$ colocado no lugar da agulha e ancorado à sínfise púbica com fio linho 3-0.

$\mathrm{O}$ laparoscópio de $0^{\circ}$ foi acoplado à câmera e a fonte de luz e introduzido na cavidade abdominal. A punção dos dois trocáteres foi feita sob visualização desta câmera.

O segundo trocáter de $5 \mathrm{~mm}$ foi colocado por uma incisão de $0,5 \mathrm{~cm}$ abaixo do apêndice xifóide sendo observado através do laparoscópio (Figura 1).

O terceiro trocáter puncionou a cavidade através de uma incisão de $0,5 \mathrm{~cm}$ no hipogástrio do animal inferiormente ao primeiro trocáter cerca de $3 \mathrm{~cm}$, obedecendo a triangulação (Figura 2).

Uma pinça "Maryland" foi colocada no último trocáter para afastar as alças intestinais e localizar o baço. Através do segundo trocáter, uma pinça atraumática elevou o baço a fim de oferecer melhor exposição das estruturas do hilo. O ligamento gastroesplênico foi dissecado e os vasos curtos gástricos foram cauterizados (Figura 3 ).

$\mathrm{O}$ órgão é elevado pela pinça atraumática e os vasos hilares superiores, centrais e inferiores foram delicadamente dissecados e cauterizados utilizando a pinça "Maryland" e o "Hook" ou gancho (Figura 4).

Para retirada do órgão foi adaptado um "endobag" de $3 \mathrm{~cm}$, cortando-se o dedo indicador de uma luva cirúrgica de tamanho médio. O "endobag" foi pinçado, acoplado ao redutor e colocado às cegas pelo trocáter de $11 \mathrm{~mm}$, de onde se retirou momentaneamente a câmera.
Retomada a posição do laparoscópio, a peça foi colocada dentro do "endobag", (Figura 5) e o conjunto foi retirado da cavidade abdominal, através da ampliação de um dos sítios dos trocáteres de $5 \mathrm{~mm}$.

Depois da retirada do órgão, a cavidade abdominal foi inspecionada quanto a possíveis focos hemorrágicos que foram hemostasiados por eletrocoagulação. Os trocáteres foram retirados sob a visão bidimensional do laparoscópio e a parede abdominal foi suturada em dois planos.

\section{Pós-operatório}

Os animais foram recolocados em gaiolas individuais e, observados durante oito dias, nas mesmas condições já citadas (ver método). Após um período de observação de oito dias os animais foram sacrificados em uma câmara fechada com éter etílico a $50 \%$ e submetidos a necrópsia.

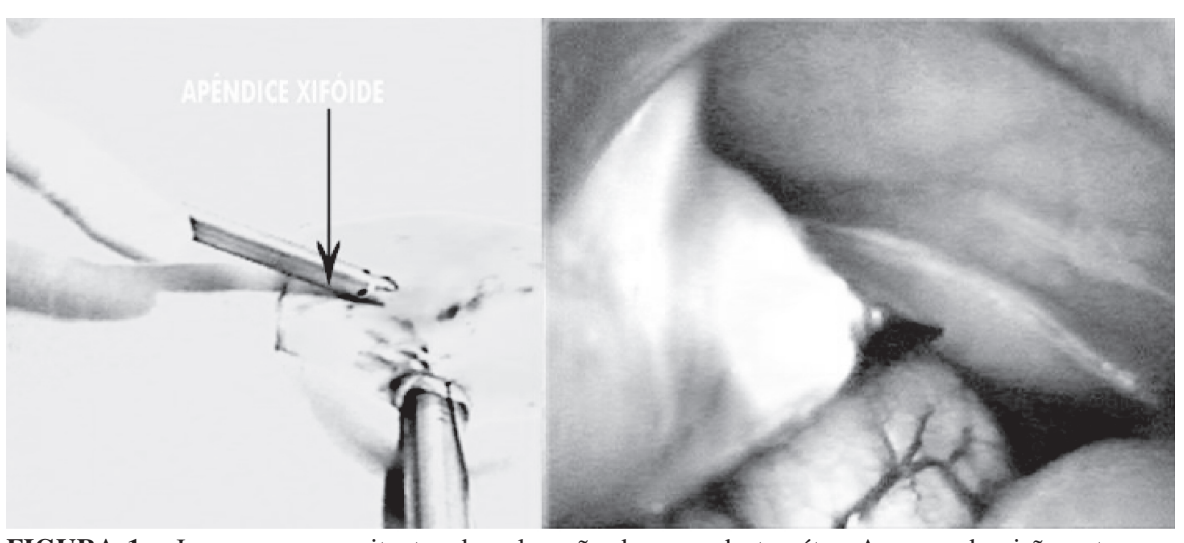

FIGURA 1 - Imagens concomitantes da colocação do segundo trocáter. A esquerda visão externa e a direita visão interna.

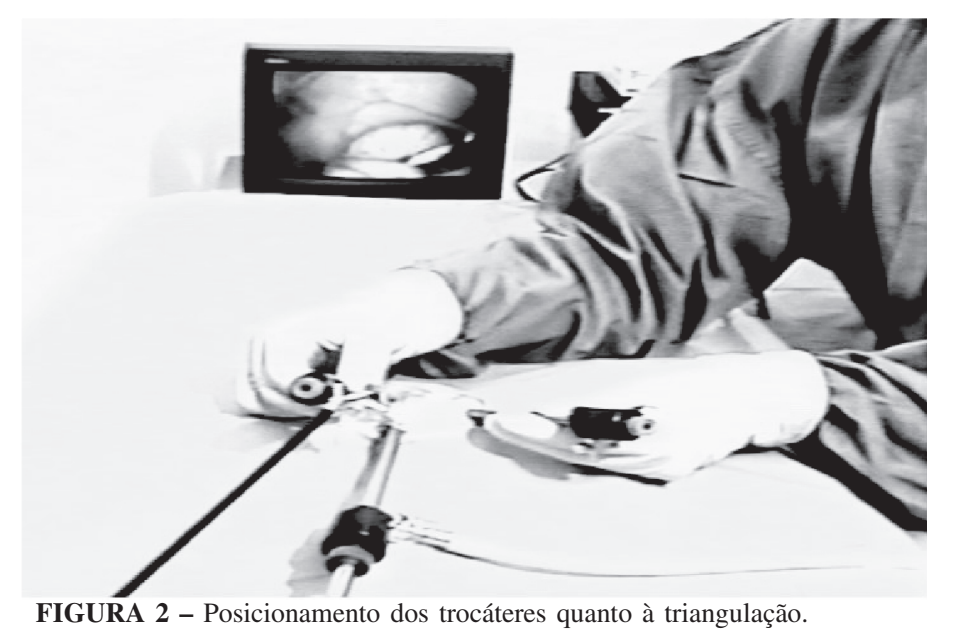

554 - Acta Cirúrgica Brasileira - Vol 18 (6) 2003 


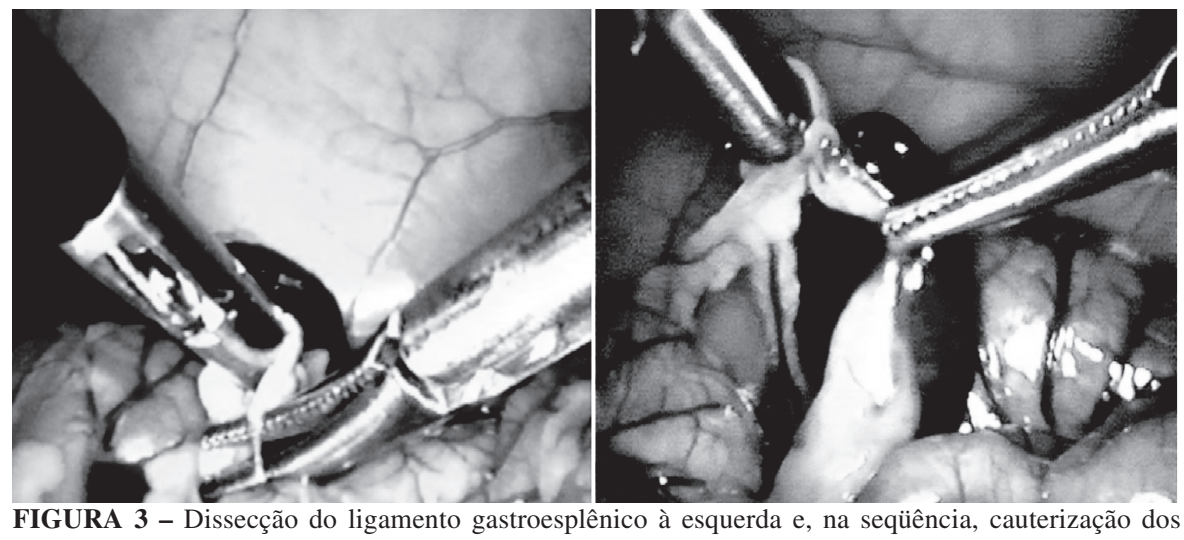

vasos curtos do estômago.

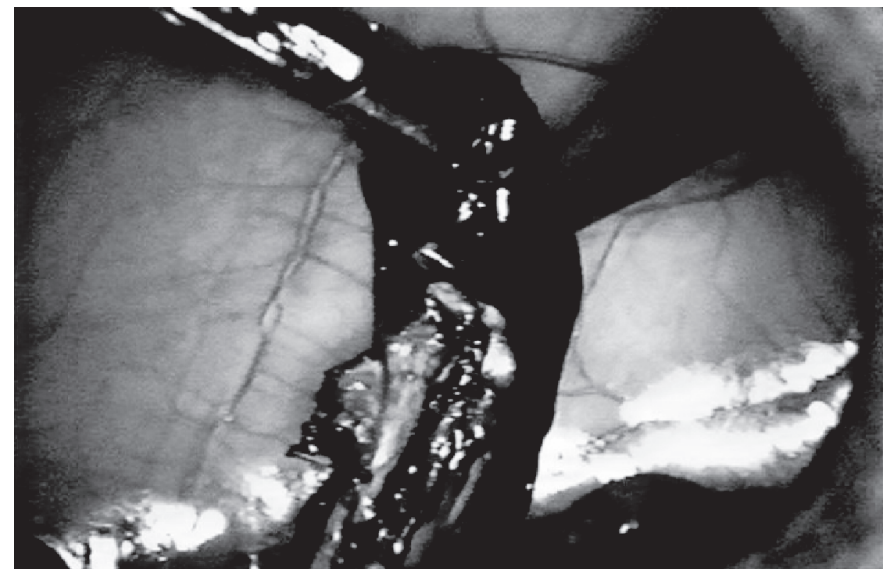

FIGURA 4 - Visão laparoscópica da dissecção completa do hilo esplênico com o "Hook".

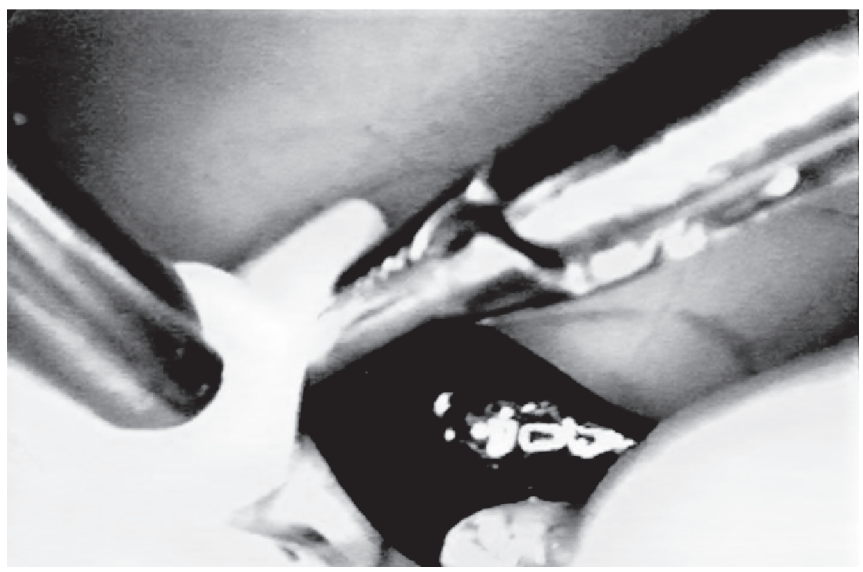

FIGURA 5 - Colocação da peça em "endobag" artesanal para retirada da cavidade.

\section{Resultados}

As esplenectomias laparoscópicas foram realizadas em média de $40+/-8$ minutos e não houve necessidade de conversão para técnica aberta. A taxa de letalidade durante a indução anestésica foi de $2,86 \%(n=1)$. Os outros 34 animais estavam vivos após o término do procedimento e as hemorragias no intraoperatório foram facilmente controladas com o eletrocautério. No decorrer do período de observação de 8 dias, quatro animais morreram (três no segundo dia e um terceiro dia), nesses animais foram encontradas hemorragias intr abdominais , com taxa de mortalidade no pósoperatório imediato de $11,42 \%$.

Ao término do período de observação a taxa de sobrevida foi de $85,71 \%$ $(\mathrm{n}=30)$. No oitavo dia de observação, a média de perda de peso foi de $10,30 \mathrm{~g}$ (desvio padrão de +/-6,84 g). Nas necropsias realizadas foram encontrados nove animais com áreas de aderência $(25,71 \%)$ envolvendo estômago e intestino. Em nenhum dos animais foram observados abscessos, hemorragia intra-abdominal ou deiscências de suturas.

\section{Discussão}

Atualmente, tem sido significante o aumento do número de procedimentos laparoscópicos capazes de oferecer aos pacientes poucas complicações operatórias, menor tempo de hospitalização, menor desconforto no pós-operatório, menor tempo de reabilitação e melhor resultado estético. A despeito desses benefícios ainda há dúvidas sobre o tratamento laparoscópico de doenças malignas e hematológicas. ${ }^{1,8,12}$. Por conta das limitações nos ensaios clínicos, é necessário um modelo animal adequado para estudos nos aspectos oncológicos e imunológicos ligados à cirurgia laparoscópica. ${ }^{8}$

$\mathrm{O}$ modelo murino apresentado se mostrou eficiente para o treinamento da cirurgia laparoscópica por possibilitar o cirurgião trabalhar num campo diminuto, o que requer um refinamento da sua técni- ca. É claro que inicialmente,o treinamento deve começar em simuladores para possibilitar o manejo com o instrumental. Passada essa fase inicial, o cirurgião já pode desenvolver sua habilidade em ratos que são animais de fácil manejo, baixo custo, boa tolerância a procedimentos cirúrgicos e são os mais estudados fisiologicamente. ${ }^{4}$ Além disso, esse modelo experimental pode ser reproduzido para estudos sobre o impacto da técnica laparoscópica na imunologia, nas doenças hematológicas e oncológicas ${ }^{15}$.

Este modelo de esplenectomia apresentou uma taxa de mortalidade compatível com a discrição do modelo Giuffrida e col. ${ }^{10}$ no qual, apesar da pequena amostra, apenas um dos seis ratos esplenectomizados morreu.

Para ser economicamente viável, este modelo de treinamento utilizou o instrumental videolaparoscópico usual da cirurgia em humanos adultos, ao contrário de outros estudos que usam mesas de contenção adaptadas ao laparoscópio, instrumental de calibre reduzido, ópticas de 
diferentes angulações, além de artroscópios e broncoscópios. ${ }^{3,4,7-11,13,14}$

Concluímos que esse modelo de esplenectomia laparoscópica em ratos é factível. As hemorragias que aconteceram no pós-operatório imediato estão de acordo com os riscos de sangramentos em estruturas ricamente vascularizadas como é o pedículo esplênico e exigem uma criteriosa revisão de hemostasia, Giuffrida e col $^{7}$ utilizaram gaze para conter o sangramento residual em seu modelo de esplenectomia o que achamos desnecessário já que o eletrocautério é eficaz e a introdução da gaze faz vazar o pneumoperitônio, perdendo campo visual e prolongando o tempo cirúrgico. Os achados de aderência podem ser explicados pela eletrocoagulação dos vasos esplênicos e pela contigüidade entre o pedículo esplênico e os outros órgãos do quadrante superior direito como estômago, intestino e rim direito.

A taxa de incidência das aderências intra-abdominais foi menor daquelas descritas na literatura, provavelmente porque os outros modelos ${ }^{3,7,10}$ utilizaram "endoloop" para ligar o pedículo vascular. Optamos por preterir esse instrumento neste procedimento por conta da proximidade entre a cauda do pâncreas e o hilo esplênico e que, através de uma ligadura, favorecêssemos a formação de abscessos pancreáticos como descreveu Guiffrida e col. ${ }^{7}$ em seu modelo experimental
Por conta do risco de sangramento no pós-operatório imediato, sugerimos um período de treinamento prévio em simuladores e um maior cuidado na manipulação de estruturas extremamente vascularizadas e delicadas de pequenos animais.

\section{Conclusão}

A esplenectomia laparoscópica em ratos é possível e pode ser utilizada em pesquisas e treinamento da técnica laparoscópica.

\section{Referências}

1. Glasgow RE, Mulvihill SJ. Laparoscopic splenectomy. World J Surg 1999;23:384-8.

2. Reyes MSV, Kalach AC, Baro $\mathrm{CH}$, Hidalgo CO. Esofagomiotomia: tecnica abierta versus laparoscópica: modelo experimental piloto en rata Wistar. An Med Assoc Med Hosp ABC 1998;43(3): 101-4.

3. Kalach AC, Baro $\mathrm{MCH}$, Vorero $\mathrm{NH}$, Marcovich DL, Rojas AC, Barrera RC, Chaba SS. La rata como modelo animal para la enseñanza e investigación de la cirugía laparoscópica. Cir Gen 1996;3: 221-7.

4. Sandoval BA, Sulaiman TT, Robinson AV, Stellato TA. Laparoscopic surgery in a small animal model: a simplified technique of retroperitoneal dissection in the rat. Surg Endose 1996;10:925-7.

5. Berguer R, Cornelius T, Dalton M. The optimum pneumoperitoneum pressure for laparoscopic surgery in the rat model. Surg Endosc 1997;11:915-8.

6. Dalton M, Hildreth J, Matsuoka T, Berguer R. Determination of cardiorespiratory function and the optimum anesthetic regimen during laparoscopic surgery in the rat model. Surg Endosc 1996; 10:297-300.

7. Gutt CN, Riemer V, Brier C, Berguer R, Paolucci V. Standardized tchnique of laparoscopic surgery in the rat. Dig Surg 1998; 15:135-9.

8. Berguer R, Gutt CN, Stiegmann GV. Laparoscopic surgery in the rat: description of a new technique. Surg Endosc 1993;7:345-7.

9. Berguer R, Gutt CN. Laparosccopic colon surgery in a rat model: a preliminar report. Surg Endosc 1994;8:1195-7.

10. Giuffrida MC, Marquet RL, Kazemier G, Wittich P, Bouvy ND, Bruining HAS, Bonjer HJ. Laparoscopic splenectomy and nephrectomy in a rat model: description of a new technique. Surg Endosc 1997; 11:491-4.

11. Szold A, Sagi B, Merhav H, Klusner JM Optimizing laparoscopic splenectomy: techical details and experience in 59 patients. Surg Endosc 1998;12:1078-81.

12. Berguer R, Alarcon A, Feng S, Gutt C. Laparoscopic cecal ligation and puncture in the rat: surgical techinique and preliminary results. Surg Endosc 1997;11:1206-8.

13. Kalach A C, Marcovich L D, Baro $\mathrm{MCH}$, Obregón PO, Medres MG, Jarcro NH, Chaba SS, Rojas AC. Microcirurgia videoscópica técnica asistida aplicada al diseño de procedimientos diversos utilizando a la rata como modelo experimental. An Med Assoc Mad Hosp ABC 1998;43(1): 5-9.

14. Lee WS, Gleason NR, Bessler M, Whelan RL. Peritoneal irrigation with povidineiodine solution after laparoscopic-assisted splenectomy significantly decreases porttumor recurrence in a murine model. Dis Colon Rectum 1999;42(3):319-26.

Costa VA, Oliveira FM, Oliveira Jr LC, Carreiro MC, Guimarães P. Na experimental model of laparoscopic esplenectomy in rats. Acta Cir Bras [serial online] 2003 Nov-Dec;18(6). Available from URL: http://www.scielo.br/acb.

ABSTRACT - Purpose: To describe a model of laparoscopic esplenectomy in rats. Methods: Thirty-five male rats Rattus Norvegicus Albinus Wistar weighing $250+/-50 \mathrm{~g}$ were used in this study. The animals were insufflated with $\mathrm{PCO}_{2}$ of $7 \mathrm{mmHg}$ through a Veress needle. After the pneumoperitoneum, two $5 \mathrm{~mm}$ and a $11 \mathrm{~mm}$ trocars were passed through the abdominal wall, so as to form a triangular geometrical figure. The spleen was approached directly by dissection of the gastrosplenic ligament. Hilar vessels were blunty dissected close to the spleen using Maryland and Hook. A bag was then introduced into the abdominal cavity and the spleen slipped inside. The organ could be removed through a port of site. Results: One animal $(2,86 \%)$ died at the anaesthetic induction. After an eight-day observation period, 30 animals survived $(85,7 \%)$ and four $(11,42 \%)$ died immediately after the surgery. In relation to complications, nine rats showed adhesions in left upper quadrant. Conclusions: This laparoscopic splenectomy is feasible and it is an excellent training model. Besides low cost and easy availability, the rat model requires less financial efforts.

KEY WORDS - Laparoscopic surgery. Splenectomy. Rat.

Correspondência:

Vanessa Alves Costa

Rua Professor Sabino Silva, 282/601

40155250 Salvador - BA

nessa@lognet.com.br
Conflito de interesse: nenhum Fonte de financiamento: nenhuma

Data do recebimento: 25/08/2003

Data da revisão: 19/09/2003

Data da aprovação: 02/10/2003 